\section{EGAS Conference}

\section{Torun, Poland / 10-13 July 1991}

The 23rd annual meeting of the European Group for Atomic Spectroscopy (EGAS) was held this year in Torun, Poland, on 10-13 July. Originally planned for Vilnius, Lithuania, the EGAS Board decided in the spring to shift the meeting because of the unpredictable political situation. In spite of the short notice, the local organizers, S. Legowski and his coworkers, with the help of the organizers from Vilnius, $Z$. Rudzikas and coworkers, were able to complete all the necessary arrangements. More than 170 scientists and students from 19 different countries came together at Nicolas Copernicus University and benefited from the informal style and relaxing atmosphere.

The scientific programme consisted of 11 invited lectures and about 120 contributions, some of which were presented orally, while the majority was given as posters. The invited lectures, most of them in a tutorial style, emphasized highlights in recent results in atomic physics, their impact on different areas of physics as well as future developments.

The conference began with a review by B.W. Petley (NPL, UK) of the present status of the system of fundamental constants, where accurate comparisons provide strin gent tests of our knowledge of the physical world. Some of the relevant measurements are firmly spectroscopic and are now approaching the $10^{-10}$ level of precision. The progress may, in some cases, end when,

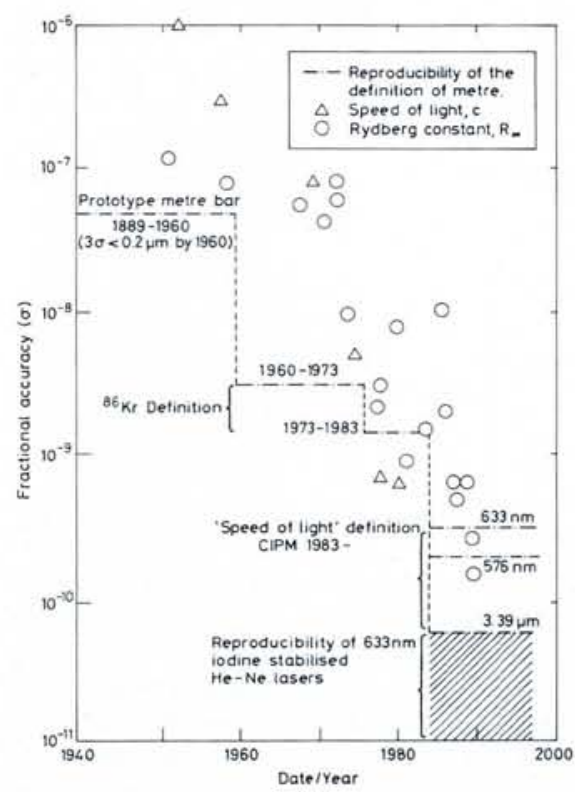

Fig. 1 - The accuracy of measurements of the Rydberg constant, of the speed of light and of the reproducibility of the metre during this century (Courtesy B.W. Petley).



Fig. 2 - Experimental setup for an atomic interferometer to record optical Ramsay fringes in a calcium atomic beam by means of four travelling waves (Courtesy F. Riehle et al., Proc. 10th Int. Conf. on Laser Spectroscopy).

e.g., weak interaction comes into play or QED effects from the conducting surfaces of the apparatus shift spectral lines. Petley pointed out the lack of precision and the need for further improvements in some other cases such as Avogadro's constant and the proton magnetic moment. Evolution in the accuracy of measurements of the Rydberg constant, of the speed of light and of the reproducibility of the metre is illustrated in Fig. 1.

W. Ertmer (Bonn, Germany) reviewed techniques to cool, focus and manipulate atomic beams by laser light. Atomic beams of very low velocity, small velocity spread and very high collimation offer further improvements to both spectroscopic measurements and fundamental investigations. One area is interferometry using cold atoms - a topic also reviewed by J. Helmcke (PTB Braunschweig, Germany) who showed that an atomic beam excited in resonance by four travelling laser wave fields in an optical Ramsey geometry (Fig. 2) acts as an atomic interferometer, and that shifts of the fringes under rotation of the interferometer may be interpreted in terms of the Sagnac effect known from optics. Rectified dipole forces in standing wave laser fields, another aspect of light-atom interaction, was discussed by A. Sidorov (Troisk, USSR).

The complexity of the emission and absorption spectra of hot plasmas (unresolved transition arrays) has revealed a need for new theoretical approaches to interpret observations. C. Bauche-Arnoult (Orsay, France) gave a number of examples to illustrate the different parameters which can be used to describe groups of lines in place of individual features and, as a second step, the application of statistical arguments to describe the shapes of some contributions.

A review of model studies on threshold phenomena in photodetachment and photoionization (J. Zaremba, Torun) presented a detailed non-perturbative description of photoionization as well as typical approximations and their limitations.
B.G. Wybourne (Canterbury, New Zealand) discussed a technologically advanced ring laser with a supercavity, having a finesse of 100000 and being able to ultimately observe frequency differences $\delta \mathrm{f} / \mathrm{f}$ of $7.10^{-20}$. Applications of such a device include detecting the earth's rotation to about $10^{-14} \mathrm{rad} / \mathrm{s}$, the investigation of nonlinear optical effects giving rise to phase shifts of only $10^{-10} \mathrm{rad}$, and the observation of optical activity induced in the vacuum by electric and magnetic fields or in an atomic gas by Coriolis effects.

Knystautas (Laval University, Quebec) described the application of atomic physics to plasma physics. Fluorescence from $\mathrm{N}$ II or O II spectra induced by the impact of 10 $\mathrm{keV} / q \mathrm{Ar}^{9+}$ ions in a Tokamak plasma allows one to monitor purification of the plasma by boron, enabling substantial progress in the performance and control of high temperature plasmas.

D. Stacey (Oxford, UK) spoke on weak interaction effects in atomic physics. $\mathrm{He}$ stressed, in particular, that experiments to detect changes in the optical rotation induced by parity violating weak currents (PNC) at the $10^{-6}$ level place enormous demands on our knowledge of systematic effects as well as requiring an atomic theory of the same level of precision, thus pushing both experimental and theoretical atomic physics to their limits. Fig. 3 gives an example of the status of our understanding of complex spectra. He summarized the results of many different experiments on different atomic systems to show that theory and experiment, after several years of discussion and continuous improvement, now agree very well. Also, the extracted value of $\sin ^{2} \theta_{w}$ via the standard model of the electroweak interaction, agrees with the values obtained from high energy experiments. Since the prediction of atomic PNC effects are very sensitive to the mass of the Z-boson, any discrepancy would imply phy-

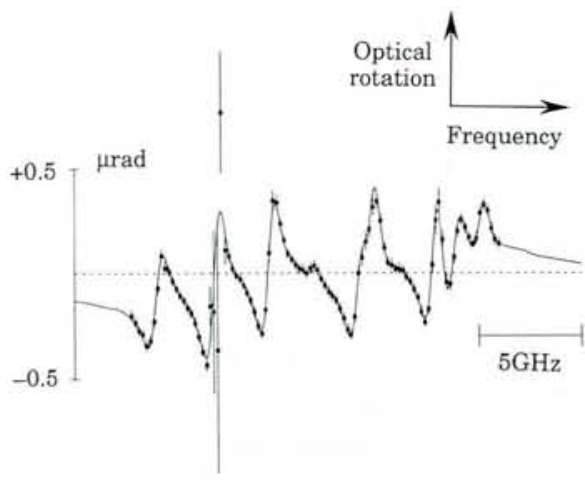

Fig. 3 - Optical rotation measured in the vicinity of the $876 \mathrm{~nm} \mathrm{M1} \mathrm{transition} \mathrm{in}$ atomic bismuth. The dominant effect is the nuclear spin-independent PNC interaction, which consists of a series of dispersionshaped curves, one centred on each hyperfine component. The signal/noise ratio is poorest near the component centres because these are the regions of high absorption. The continuous line is the best fit theoretical curve (Courtesy M.J. Macpherson et al., submitted to Phys. Rev. Lett.). 
sics beyond the standard model, e.g., extra heavy bosons.

The general behaviour and recent results of spectroscopic experiments using laser cooled highly charged ions travelling at high speed in a storage ring at GSI, Darmstadt, were presented by Th. Kühl. Different velocity classes of a circulating $\mathrm{Li}^{+}$beam can be excited by lasers (Fig. 4) and an experiment involving a precision test of special relativity is underway.

A fascinating and rapidly developing field deals with the properties and applications of squeezed states of light, where the uncertainty of the photon number in a light beam is reduced at the expense of phase fluctuations. H.A. Bachor (Canberra, Australia) discussed experimental setups to produce such states and to improve the degree of squeezing, pointing out that perhaps the most interesting applications for squeezed light might perhaps not have been imagined so far.

The large number of contributions represented an impressive demonstration of the general status of atomic spectroscopy, our ability to deal with very complex spectra, the improved instrumentation, and prospects for further refinements of experiments to reveal new and subtle features of atomic spectra.

\section{E. Biémont}

Université
de Liège
Universität

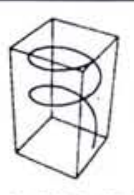

\section{Mathematica}

$\mathrm{TM}$

A System for Doing Mathematics by Computer

A Wolfram Research Inc. product

$\square$ Numerics - Works with numbers of arbitrary magnitude and precision.

$\square$ Symbolics - Encyclopaedia of mathematical functions and operations used in arithmetic, algebra and analysis.

Procedural, functional and mathematical programming.

$\square$ Graphics - 2D, 3D and animated PostScript graphics.

$\square$ Text processing - Fully interactive reports and textbooks.

$\square$ Runs on - MS-DOS based computers; Macintosh, Apollo, Hewlett Packard, IBM AIX/RT, MIPS, Silicon Graphics, Sony, Sun, VAX.

Now available in Europe from:

MathSoft Overseas, Inc.

POB 641, 1211 Geneva 3, Switzerland Tel. ++41 (22) 465260

Fax $++41(22) 465939$

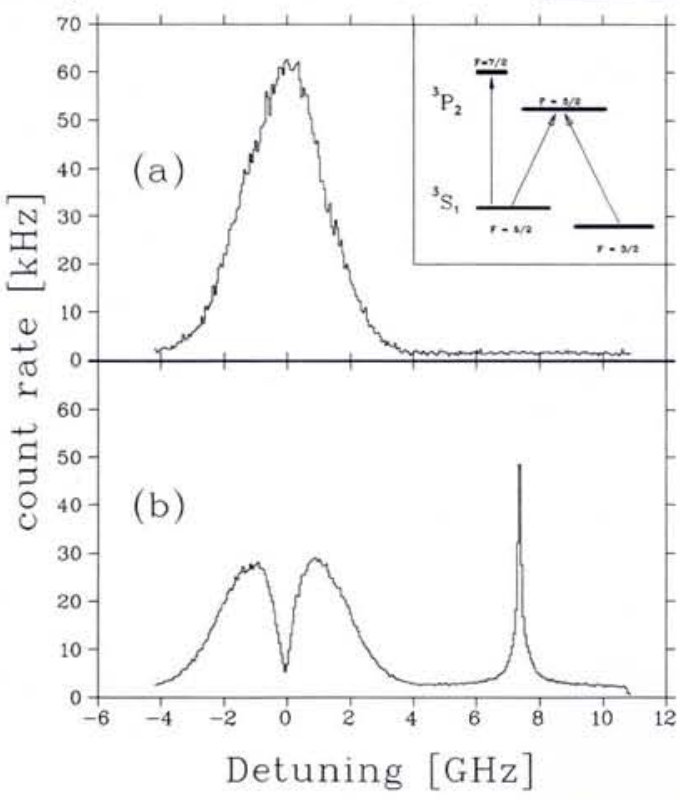

Fig. 4 - Excitation of a $13.3 \mathrm{MeV} \mathrm{Li}^{+}$beam in the Heidelberg Test Storage Ring by two counterpropagating laser beams at 585 and $514 \mathrm{~nm}$ co- and counter-linear with the ion beam. The frequencies are chosen such that the Doppler shifted frequencies match the restframe transitions. Trace (a) shows the initial longitudinal momentum distribution of the $\mathrm{Li}^{+}$beam probed by the ${ }^{3} S,(F=5 / 2)-{ }^{3} P_{2}(F=7 / 2)$ transition. A dip in the distribution appears when the second resonance is set on the ${ }^{3} S,(F=5 / 2)-$ ${ }^{3} P_{2}(F=5 / 2)$ transition in resonance with the ions in the centre of the distribution. The excited ions, being pumped into the $F=3 / 2$ state, reappear with a narrow velocity spread in the laser induced fluorescence spectrum when one of the lasers is detuned to the ${ }^{3} S,(F=3 / 2)-{ }^{3} P_{2}(F=5 / 2)$ transition (trace $b$ ) (Courtesy Th. Kühl).

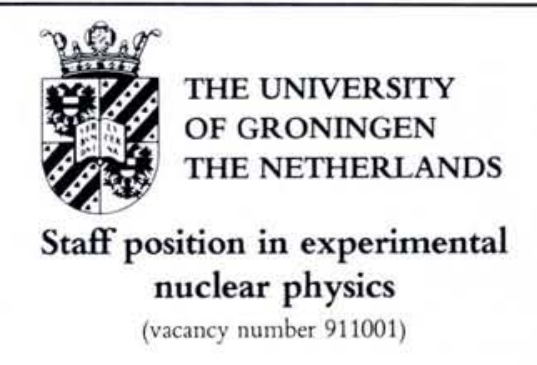

The KVI is a Dutch national institute for Nuclear Physics research. In 1994 a new superconducting cyclotron, AGOR, will be available, which will accelerate heavy ion beams up to $100 \mathrm{MeV}$ / nucleon and protons and deuterons to a maximum energy of $200 \mathrm{MeV}$. The institute foresees the installation of a magnetic spectrograph and a source for polarized protons and deuterons.

We have an opening for a new staff member in the group, responsible for setting up this source and for initiating a research program, which will emphasize the study of fundamental symmetries and interactions.

The candidate should preferably have postdoctoral experience. He will contribute to the teaching program.

Salary max. Hfl. 7.528,- per month.

Send applications before December 1st 1991 to Ms. A.M. van der Woude, KVI, 9747 AA Groningen, the Netherlands, with a curriculum vitae, a list of publications and the names of three references.

Further information can be obtained from Prof. Dr. R. Malfliet or Dr. S.Y. van der Werf, telephone resp. $31-50-633554$ or $31-50-633620$. Telefax 31-50-634003.

\begin{tabular}{l}
\hline MANAGING EDITORS \\
\hline Geoffrey C Fox (Syracuse Univ.) \\
Hans J Herrmann (HLRZ, Jülich) \\
Kunihiko Kaneko (Univ. of Tokyo) \\
The scope of this journal covers Computa- \\
tional Physics, Physical Computation and
\end{tabular}
related subjects. IJMPC aims to publish both review and research articles on the use of computers to advance knowledge in the physical sciences and the use of physical analogies in computation. Some topics to be included are computer algebra, numerical simulation techniques, parallel and vector computers, lattice gauge theory, algorithms, etc.

\section{SOME PAPERS}

- Simulations of Kinetic Roughening with Power-Law Noise of the Connection Machine (R Bourbonnais, H J Herrmann \& T Vicsek)

- Computational Ecosystems in a Changing Environment (N Glance, T Hogg \& B A Huberman)

- Parallelization of Short-and LongRange Cellular Automata on Scalar, Vector, SIMD and MIMD Machines

- High Speed Simulations of Ordered and Disordered Cellular Automata ( $R$ W Gerling \& D Stauffer) (G A Kohring)



SUBSCRIPTION INFORMATION ISSN: 0129-1831 Individuals/Institutions/Libraries US\$220 (airmail please add US\$25) Special Rates areavailable to individuals/Institutions/Librariesfrom developing countries. Please write in for details. 